\title{
Knowledge Management for Change Prediction by the Integrated Multi- disciplines: Computational Intelligence, Advanced Software Engineering, and Statistics
}

\author{
Author: Shin-Jye Lee ${ }^{1}$; Ching-Hsun Tseng ${ }^{2}$; Hsueh-Cheng Liu ${ }^{1}$ \\ Affiliation: Institute of Management of Technology, National Chiao Tung University, Hsinchu, \\ Taiwan $^{l}$; Department of Computer Science, University of Manchester, United Kingdom ${ }^{2}$
}

E-mail: camhero@gamil.com ${ }^{1}$; hank131415go61@gmail.com ${ }^{2}$;stdm11528@gmail.com³

DOI: 10.26821/IJSHRE.9.3.2021.9317

\begin{abstract}
This proposal proposes a multi-disciplinary methodology integrating Computational Intelligence (Fuzzy Theory and Genetic Algorithm), Advanced Software Engineering (Theory of Stream $X$-Machine and the procedure of Advanced Software Engineering), Calculus (the method of Least Squares) and Statistics (Simple Linear Regression Model). Meanwhile, the proposed methodology can not only overcome the drawbacks of inadvertently ignored problems, but also can make a higher performance of this achievement.
\end{abstract}

Keywords: Knowledge Management, Change Prediction, Computational Intelligence

\section{INTRODUCTION}

Being reliable, Knowledge Management is one of popular subjects applied at various fields in the industrial engineering, and it has been a prevalent methodology in industry management as well. With the improvement of Knowledge Management, Knowledge Management for change prediction has also been emphasized on and innovatively developed nowadays. To get a higher achievement, more complex products are trying to design better than original one by changing existing designs. However, sometimes these changes can be problematic because of negligence during the process on designing. Therefore, how to develop change management methods and apply them as a foundation to find the positive pattern about past changes and potential problems become increasingly important issues. There exit several ways to improve the problem and make the achievement optimal, and one of which is work it out by integrating multi-disciplines. This research aims to develop change management methods with Computational Intelligence, Advanced Software Engineering, Calculus and Statistics. In order to try and understand what are at stock, I'll focus my research on the following main topics.

\section{COMPUTATIONAL INTELLIGENCE}

Computational Intelligence is the study of the design of the intelligent agents [1]. An agent can be recognized as an approach or something which can work the system effectively in a variety of disciplines, including pure science, social science, medical science and engineering. Also, an intelligent agent means it can work intelligently [1]. Nowadays, there are so many fields and theories trying to carry Computational Intelligence out, such as Machine Learning, Data Mining, and Fuzzy Theory. Moreover, Fuzzy Theory and Genetic Algorithm will play two important roles in this project. 
Volume 9 Issue 3 March 2021

\section{ADVANCED SOFTWARE} ENGINEERING

\subsection{The procedure of Advanced Software Engineering}

In Advanced Software Engineering, as shown in Fig. 1, the procedure is a recursive circle. Meanwhile, the procedure is made up of seven steps, including feasibility, requirement analysis, general design, detailed design, implementation, testing and new requirement.

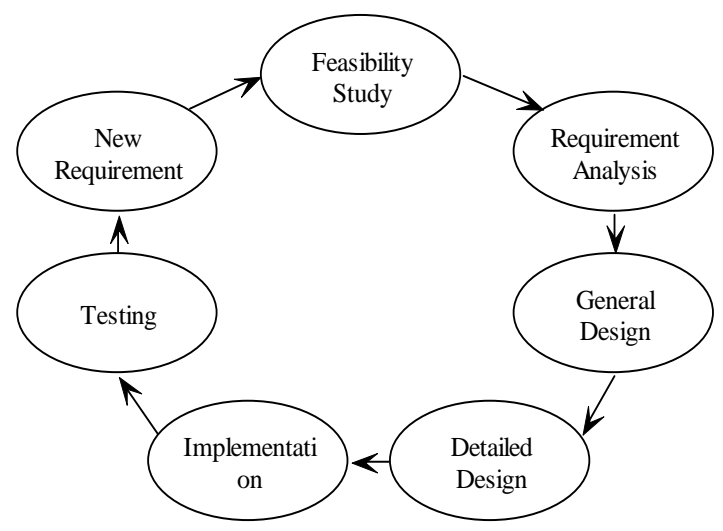

Fig 1: The procedure of Advanced Software Engineering

\subsection{Stream X-Machine}

In 1974, Eilenberg [2] introduced a powerful theory generalized from Finite State Machine, and the concept of X-Machine is used for Nerification and testing. Basically, the concept of X-Machine is evolved from the theory of Finite State Machine, but the theory of X-Machine is more powerful than that of Finite State Machine. In 1995 Ipate [3] developed a type of X-Machine - the theory of Stream X-Machine modified form X-Machine. Also, X-Machine can detect faults more precise than Finite State Machine, because X-Machine contains memory and a set of data processing functions more. However, Stream X-Machine can not only get the same power with X-Machine, but also works easier than X-Machine.

\section{RESEARCH METHODOLOGY}

Multi-disciplinary theories will be worked and integrated into one methodology. In addition to Computational Intelligence, this methodology is also carried out by the procedure of Advanced Software Engineering, Calculus and Statistics. In this methodology, the procedure of Advanced Software Engineering will be refined by computational intelligence. Therefore, the procedure can be described as follows:

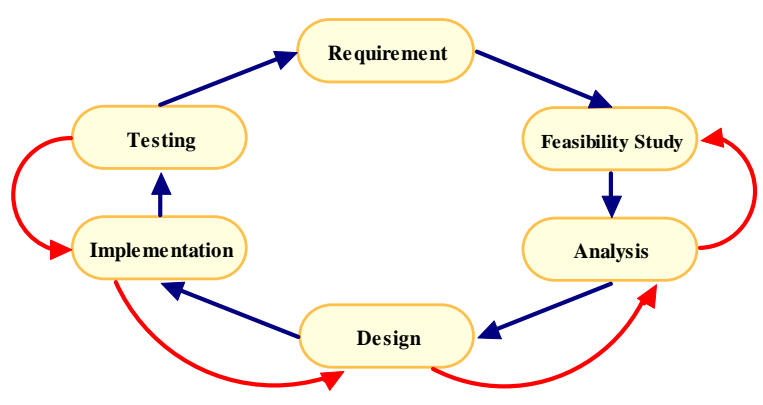

Fig 2: The proposed procedure of Knowledge Management for Change Prediction by Multidisciplines. Blue line-direct route, Red linereturn route.

\subsection{Feasibility Study}

Literature survey and requirement consideration will be mainly worked at early stage of the research to find out the feasibility. To understand the detail of the requirement and the possibility of the achievement, not only the requirement of a project will be fully understood, but even key design of a project will also be thoughtfully understood by Feasibility Study. Therefore, Feasibility Study is the first step in the procedure, and it plays an essential role in the whole methodology.

\subsection{Analysis}

Computational Intelligence will be mainly worked as an effective assistant in this step. Fuzzy Theory, Genetic algorithm, the method of Least Squares or something about Computational Intelligence will be used here. In the light of information worked by the first step - Feasibility Study, information will be fuzzified and then come Fuzzy rules out by Fuzzy Theory, and then generate weighted Fuzzy rules by Genetic Algorithms or the method of Least Squares. Final, Simple Linear Regression Model will come out the best value or parameter. Here provides two types of methods, and the simply regular steps are described in Fig. 3 and Fig. 4:

- $\quad$ Method 1

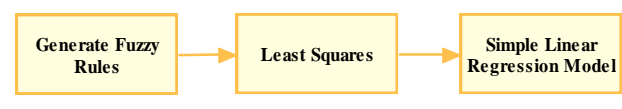

Fig 3: The procedure of Methodology I. 
Volume 9 Issue 3 March 2021

- $\quad$ Method II

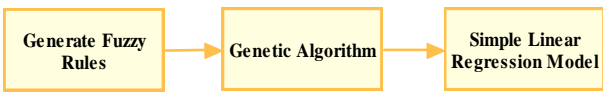

Fig 4: The procedure of Methodology II.

\subsection{Design}

This step, in accordance with the attributes or values computed by the step 2 - Analysis, designs the module or function of products.

\subsection{Implementation}

This step, in accordance with the design by the step 3 - Design, implements the module or function of products.

\subsection{Testing}

Testing is a very significant step in the whole procedure, because faults can be detected by Stream X-Machine immediately. Meanwhile, inadvertently ignored problems may be detected in this step. Definitely, this step is mainly worked for finding faults and minimizing errors effectively.

As a whole, in addition to "Requirement" step and "Feasibility" step, each step of the procedure can return to the previous step, in case something wrong occurs occasionally.

\section{CONCLUSION}

This proposal presents an innovative methodology trying to work this project out, integrating Computational Intelligence, the method of Least Squares, Statistics and Advanced Software Engineering. Certain theories of Computational Intelligence, Calculus, Simple Linear Regression Model and the regular procedure of Advanced Software Engineering are worked in this research, and Analysis step as well as Testing step in particular. However, there are still quite a number of methods that can be discovered, and which lead to their unique results. When the methodology works as the base of this research proposal, the new requirements or ideas may be discovered in the future. Basically, the methodology runs recursively until an approximate optimization has been generated, and the coming advanced or relevant researches may be discovered as the base of this research proposal in the future.

\section{Reference}

[1] Poole, D., Mackworth, A. and Goebel, R., (1998), Computational intelligence: a logical approach, New York; Oxford: Oxford University Press.

[2] Eilenberg, S., (1974), Automata, Languages and Machines, Academic Press.

[3] Ipate, F., (1995), Theory of X-Machines and Applications in Specification and Testing, $\mathrm{PhD}$ thesis, University of Sheffield Department of Computer Science. 